\title{
$\alpha_{1 \mathrm{~A}}$-Adrenergic Receptor-Directed Autoimmunity Induces Left Ventricular Damage and Diastolic Dysfunction in Rats
}

\author{
Katrin Wenzel ${ }^{1,2 *}$, Gerd Wallukat ${ }^{2}$, Fatimunnisa Qadri ${ }^{1}$, Norbert Hubner ${ }^{2}$, Herbert Schulz ${ }^{2}$, Oliver \\ Hummel $^{2}$, Florian Herse', Arnd Heuser ${ }^{2}$, Robert Fischer ${ }^{1,2}$, Harald Heidecke ${ }^{3}$, Friedrich C. Luft ${ }^{2,4}$, \\ Dominik N. Muller ${ }^{2}$, Rainer Dietz ${ }^{1}$, Ralf Dechend ${ }^{2,4}$
}

1 Medical Faculty of the Charité, Berlin, Germany, 2 Experimental and Clinical Research Center and Max-Delbrück Center for Molecular Medicine, Berlin, Germany, 3 CellTrend, Luckenwalde, Germany, 4 HELIOS Clinic-Berlin, Franz-Volhard Clinic, Berlin, Germany

\begin{abstract}
Background: Agonistic autoantibodies to the $\alpha_{1}$-adrenergic receptor occur in nearly half of patients with refractory hypertension; however, their relevance is uncertain.

Methods/Principal Findings: We immunized Lewis rats with the second extracellular-loop peptides of the human $\alpha_{1 A^{-}}$ adrenergic receptor and maintained them for one year. $\alpha_{1 A}$-adrenergic antibodies $\left(\alpha_{1 A}-A R-A B\right)$ were monitored with a neonatal cardiomyocyte contraction assay by ELISA, and by ERK $1 / 2$ phosphorylation in human $\alpha_{1 A}$-adrenergic receptor transfected Chinese hamster ovary cells. The rats were followed with radiotelemetric blood pressure measurements and echocardiography. At 12 months, the left ventricles of immunized rats had greater wall thickness than control rats. The fractional shortening and $\mathrm{dp} / \mathrm{dt}_{\max }$ demonstrated preserved systolic function. A decreased E/A ratio in immunized rats indicated a diastolic dysfunction. Invasive hemodynamics revealed increased left ventricular end-diastolic pressures and decreased $\mathrm{dp} / \mathrm{dt}_{\min }$. Mean diameter of cardiomyocytes showed hypertrophy in immunized rats. Long-term blood pressure values and heart rates were not different. Genes encoding sarcomeric proteins, collagens, extracellular matrix proteins, calcium regulating proteins, and proteins of energy metabolism in immunized rat hearts were upregulated, compared to controls. Furthermore, fibrosis was present in immunized hearts, but not in control hearts. A subset of immunized and control rats was infused with angiotensin (Ang) II. The stressor raised blood pressure to a greater degree and led to more cardiac fibrosis in immunized, than in control rats.
\end{abstract}

Conclusions/Significance: We show that $\alpha_{1 A}-A R-A B$ cause diastolic dysfunction independent of hypertension, and can increase the sensitivity to Ang II. We suggest that $\alpha_{1 A}-A R-A B$ could contribute to cardiovascular endorgan damage.

Citation: Wenzel K, Wallukat G, Qadri F, Hubner N, Schulz H, et al. (2010) $\alpha_{1 \mathrm{~A}}$-Adrenergic Receptor-Directed Autoimmunity Induces Left Ventricular Damage and Diastolic Dysfunction in Rats. PLoS ONE 5(2): e9409. doi:10.1371/journal.pone.0009409

Editor: Marcelo G. Bonini, University of Illinois at Chicago, United States of America

Received October 15, 2009; Accepted January 16, 2010; Published February 24, 2010

Copyright: (c) 2010 Wenzel et al. This is an open-access article distributed under the terms of the Creative Commons Attribution License, which permits unrestricted use, distribution, and reproduction in any medium, provided the original author and source are credited.

Funding: The Deutsche Forschungsgemeinschaft supported Ralf Dechend and Dominik N. Muller with grants in-aid. The Helmholtz Foundation also supported the studies. Dominik N. Muller is a Helmholtz Fellow. The funders had no role in study design, data collection and analysis, decision to publish, or preparation of the manuscript.

Competing Interests: For Ralf Dechends affiliation: HELIOS Clinic is a hospital which has an affiliation with the Max-Delbrueck Center (MDC). He has a contract where he does $60 \%$ clinical duties at the HELIOS clinic and $40 \%$ research at the Max-Delbrueck Center for Molecular Medicine at the Experimental and Clinical Research Center. He also declares that there are no conflicts of interest concerning commercial affiliations and/or patents and/or data sharing with the HELIOS Clinic and the MDC. There are no restrictions on the availability or on the use of datasets.

* E-mail: katrin.wenzel@charite.de

\section{Introduction}

$\alpha_{1}$-adrenergic receptors $\left(\alpha_{1}\right.$-AR $)$ mediate vascular smooth muscle cell (VSMC) contraction, cardiac inotropy, hypertrophy, and remodeling [1]. Others and we have described agonistic autoantibodies against the $\alpha_{1}$-AR in hypertensive patients $[2,3,4,5]$. We found earlier that $\alpha_{1}$-AR-autoantibody immunoadsorption reduced blood pressure in patients with refractory hypertension [5]. In that study, rabbit or patient-derived $\alpha_{1 \mathrm{~A}^{-}}$ AR-autoantibodies were purified with chromatography and characterized by epitope mapping and surface plasmon resonance measurements. Phospholipase A2 group IIA (PLA2-IIA) and L- type calcium channel (Cacna1c) genes were upregulated in cardiomyocytes and VSMC after stimulation with both purified antibodies from patients and from rabbit [5]. We showed that patient and rabbit $\alpha_{1 \mathrm{~A}}$-AR-antibodies result in protein kinase $\mathrm{C}$ alpha activation and transient extracellular-related kinase (ERK1/ 2) phosphorylation. The antibodies also exerted acute effects on intracellular $\mathrm{Ca}^{2+}$ in cardiomyocytes and contracted mesentery artery segments [5]. In a proof-of-concept study involving the $\beta_{1^{-}}$ $\mathrm{AR}$, Jahns et al immunized rats and showed that agonistic autoantibodies caused idiopathic dilated cardiomyopathy in the same rats. Furthermore, passive transfer also caused disease $[6]$. 
Three different receptor subtypes mediate $\alpha_{1}$-adrenergic signaling, namely $\alpha_{1 \mathrm{~A}^{-}}, \alpha_{1 \mathrm{~B}^{-}}$, and $\alpha_{1 \mathrm{D}^{-}} \mathrm{AR}$. All subtypes were expressed in cardiac tissue but differ in the amino acid sequence of the second extracellular loop. Zhou et al immunized rats with a second extracellular loop peptide from the $\alpha_{1 \mathrm{D}}-\mathrm{AR}$ subtype epitope [7]. The rats developed agonistic antibodies. Tail-cuff systolic blood pressure was not changed. The investigators described cardiac hypertrophy, increase in the collagen deposition, c-jun, and matrix metalloproteinase 2 (MMP2) expressions in the heart. We immunized our animals against the second extracellular loop of $\alpha_{1 \mathrm{~A}}-\mathrm{AR}$. This report is the first showing the in vivo relevance of $\alpha_{1 \mathrm{~A}}-\mathrm{AR}-\mathrm{AB}$ (as opposed to $\alpha_{1 \mathrm{D}}-\mathrm{AR}-\mathrm{AB}$ ) to our knowledge. We investigated the effects on blood pressure by radiotelemetry and on cardiac function by invasive hemodynamic measurements with a conductance catheter and echocardiography. Cardiac molecular pathways influenced by $\alpha_{1 \mathrm{~A}}-\mathrm{AR}-\mathrm{AB}$ signaling were investigated by gene expression array analyses. Furthermore, we tested the hypothesis whether immunized rats react more sensitive to angiotensin (Ang) II.

\section{Materials and Methods}

\section{Immunization}

Experiments were performed in 36 male Lewis rats aged 8 weeks. We prepared a synthetic GWRQPAPEDETICQINEEPGYVLFSAL-AmidxTFA/salt (Biosyntan GmbH, Berlin, Germany) peptide corresponding to the second extracellular loop of human $\alpha_{1 \mathrm{~A}}-\mathrm{AR}$. Eighteen rats were immunized by subcutaneous injection $(200 \mu \mathrm{g}$, treated with $350 \mu \mathrm{g}$ methylated albumin) dissolved in $1 \mathrm{~mL}$ saline at 0,2 , and 4 weeks. The animals were boosted monthly over 12 months. Eighteen control rats received saline. For Ang II infusion, osmotic pumps (Alzet, Cupertino, CA, USA) were implanted under isoflurane anesthesia in the animals ( $\mathrm{n}=6$ per group) 12 months after first immunization. The animals received $200 \mathrm{ng}$ Ang II $/ \mathrm{kg} / \mathrm{min}$ for 14 days (Calbiochem, La Jolla, CA, USA). Local authorities (LAGeSO, Berlin, Germany) approved the animal protocol that complied with criteria outlined by the American Physiological Society.

\section{$\alpha_{1}$-AR-AB Detection}

Rat $\alpha_{1 \mathrm{~A}}-\mathrm{AR}-\mathrm{AB}$ were detected by peptide ELISA (CellTrend, Luckenwalde, Germany). Rat sera $(100 \mu \mathrm{L}), 3$ or 12 months after first immunization, were added (dilution 1:1000). As second antibody, we used rabbit anti rat IgG fc horseradish peroxidase (HRP) conjugated (1:35000 diluted, $100 \mu \mathrm{L} /$ well, Bethyl, Montgomery, TX, USA). The reaction was detected by tetramethylbenzidine (TMB) as substrate for the enzyme HRP.

Neonatal rat cardiomyocyte contraction assay and the detection of extracellular regulated kinase $1 / 2(\mathrm{ERK} 1 / 2)$ phosphorylation in CHO cells stably transfected with human $\alpha_{1 \mathrm{~A}}-\mathrm{AR}\left(\mathrm{CHO} / \alpha_{1 \mathrm{~A}}-\mathrm{AR}\right)$ were carried out as earlier described [5]. For the ERK1/2 phosphorylation experiments, $50 \mu \mathrm{g}$ of IgG purified from sera of rats 3 months after immunization and controls were added to the $\mathrm{CHO} / \alpha_{1 \mathrm{~A}}-\mathrm{AR}$ cells for $10 \mathrm{~min}$. We checked specificity by inhibiting with $1 \mu \mathrm{M}$ of $\alpha_{1}$-AR antagonists prazosin or urapidil. The development of $\mathrm{AT}_{1}-\mathrm{AR}-\mathrm{AB}, \beta 1-\mathrm{AR}-\mathrm{AB}$, or $\beta_{2} \mathrm{AR}-\mathrm{AB}$ during immunization or Ang II treatment was excluded by cardiomyocyte contraction assay in presence of the antagonists.

\section{Echocardiography, Blood Pressure and Hemodynamic Measurements}

Rats were anesthetized with $2 \%$ isoflurane and kept warm on a heated platform. Temperature and ECG were continuously monitored. Cardiac function and morphology were assessed by echocardiography with a VisualSonics Vevo 770 High-Resolution Imaging System with the use of a high-resolution $(37.5 \mathrm{MHz}$ ) transducer. The telemetry system (Dataquest ART 4.0 ${ }^{\mathrm{TM}}$, Data Sciences International, St. Paul, MN, USA) and the implantation procedure is described in detail by Brockway et al. [8]. The radiotelemetry pressure transducers (TA11PA-C20) were implanted in the abdominal cavity of the rat under isoflurane anesthesia, with the transducer connected capillary tubing anchored in the lumen of the abdominal aorta. Before the implantation the zero offset was measured and the unit was soaked in $0.9 \% \mathrm{NaCl}$. Animals were allowed to recover for 10 days. The data from the TA11PA-C20 device were transmitted via radiofrequency signals to a receiver below the home cage and thereafter collected (sampling rate $500 \mathrm{~Hz}$ ). The system monitors mean, systolic and diastolic blood pressure, heart and respiration rate and locomotor activity at 5-min intervals, while the rats move freely. We report mean arterial blood pressure (MAP). For hemodynamic measurements, rats were intubated and ventilated under isoflurane anesthesia. A 2-French conductance catheter (SPR 838 Aria, Millar Instruments, Houston, TX, USA) was inserted into the left ventricle through the right carotid artery. The stroke volume (SV), end-diastolic volume (Vol max), end-systolic volume (Vol min), and LV-pressures were measured directly in acute experiments.

\section{Gene Expression and Immunohistochemistry}

Total RNA was extracted from the cardiac apex (one-third from the whole heart including parts of left, right ventricle and septum) of three immunized and three control rats using the RNeasy Purification Kit (Qiagen GmbH, Hilden, Germany). RNA was treated by deoxyribonuclease I (Qiagen). Two $\mu \mathrm{g}$ RNA of cells were transcribed in cRNA with One-Cycle Target labeling and Control Reagents (Affymetrix, Santa Clara, CA, USA). Non-pooled microarray experiments were performed using Rat Genome 230 2.0 Arrays (31,099 probe sets, Affymetrix). Gene expression and RT-PCR experiments were carried as previously described.[5] Primer sequences for TaqMan analyses are listed in Supplementary table S1. The MIAME-compliant microarray data are available http://www.ebi.ac.uk/arrayexpress/experiments/E-TABM-725.

Cardiac tissues were fixed in paraformaldehyde and embedded in paraffin. The $5 \mu \mathrm{m}$ thick sections were deparaffinized, rehydrated, and stained by the Trichrome-Masson-Goldner or $0.1 \%$ Sirius red saturated in picric acid. Histomorphological analysis and cardiomyocyte diameter was determined on elastica van Gieson and hematoxylin-and-eosin-stained, $4 \mu \mathrm{m}$-thick sections of tissue placed in $5 \%$ formalin. Cardiomyocyte diameter was determined perpendicular to the outer contour of the cell membrane at the nucleus level in 15 representative myocytes of the section as desribed by van Heerebeek et al [9]. Heart sections were photographed at a magnification of 10x (interstitial) or 20x (perivascular) with a Sony AVT-Horn camera using Zeiss-Axioplan 2 microscope. 25 microscopic view fields (left ventricle and septum) were evaluated. Perivascular fibrosis was analysed from cross sections of coronary arteries (12 arteries per section). Analysis and quantification of interstitial and perivascular fibrosis content were performed with "Image J" software public domaine (Wayne Rasband, NIH, USA). Data are presented as fractional area of fibrosis content in \% of myocardial tissue. The investigators performing the analysis were not aware of the experimental groups.

\section{Statistics}

For analysis of echocardiography, telemetric, and hemodynamic measurements Mann-Whitney-test was used at $\mathrm{P}<0.05$. Data are expressed as mean $\pm \mathrm{SD}$. Continuous variables (blood pressure 
and heart rate) were analyzed by repeated measures analysis of variance with appropriate corrections.

\section{Results}

\section{Immunization and $\alpha_{1 A}-A R-A B$ Detection}

The cardiomyocyte contraction assay documented an increase of $\alpha_{1 \mathrm{~A}}-\mathrm{AR}-\mathrm{AB}$ activity one month after first peptide injection persisting over the immunization process of 12 months. Urapidil inhibited the activity (Figure 1A). IgG fractions eluted from sera of control rats were negative in the cardiomyocyte contraction assay (Figure 1B). The development of autoantibodies against other receptors during immunization or Ang II treatment was excluded by cardiomyocyte contraction assay in presence of the specific antagonists. The combination of $\alpha_{1 \mathrm{~A}}-\mathrm{AR}-\mathrm{AB}$ and $\mathrm{Ang}$ II increased the cardiomyocyte contraction, compared to the effect of $\alpha_{1 \mathrm{~A}}$-AR$\mathrm{AB}$ or Ang II alone (Figure 1C). A high titer of $\alpha_{1 \mathrm{~A}}-\mathrm{AR}-\mathrm{AB}$ was detected by ELISA in the immunized rats. The controls did not show a signal (Figure 1D). The incubation of $\mathrm{CHO} / \alpha_{1 \mathrm{~A}}-\mathrm{AR}$ cells with IgG prepared from immunized rats resulted in a stronger ERK1/2 phosphorylation than IgG fractions eluted from sera of control rats (Figure 2A). The specificity was proven by inhibition with the $\alpha_{1}$-AR receptor antagonist prazosin $(62 \%$ inhibition, $\mathrm{P}=0.023$, Figure 2B).

\section{Cardiac Function in Immunized Rats}

The diastolic interventricular septum and diastolic left ventricular heart wall thickness were significantly increased in 12 months immunized rats compared to the controls (Figure $3 \mathrm{~A}$ ). The ratio of heart/body weight $(\mathrm{HW} / \mathrm{BW} \times 1000)$ was significantly increased in the immunized rats compared to the controls $(2.21 \pm 0.23$ vs. $1.97 \pm 0.04, \quad P=0.0471$, Supplementary table S2). Fractional shortening was similar in both groups, so that systolic function was preserved (Figure $3 \mathrm{~B}$ ). A decreased ratio of peak flow velocity of the early rapid diastolic filling wave to peak flow velocity of the late diastolic filling wave ( $\mathrm{E} / \mathrm{A}$ ratio) in immunized rats indicates diastolic dysfunction (Figure $3 \mathrm{C}$ ). The echocardiographic findings in vivo are summarized in Supplementary table S3. Hemodynamic measurements showed higher left ventricular end-diastolic pressures (LVEDP) in immunized rat hearts (Figure $3 \mathrm{D}$ ). No changes in $\mathrm{dp} / \mathrm{dt}_{\max }$ confirmed the preserved contractility/systolic function (Figure $3 \mathrm{E}$ ) and the decreased $\mathrm{dp} / \mathrm{dt}_{\min }$ indicates an impaired relaxation/diastolic function (Figure $3 \mathrm{~F}$ ). The analysis of the mean cardiomyocyte diameters showed a significant hypertrophy 12 months after immunization with $\alpha_{1 \mathrm{~A}}$-AR peptide, compared to controls (Figure $3 \mathrm{G}$ ). The blood pressure (MAP) and heart rate measured by telemetry were not different 12 months after first immunization (Figure 4).

\section{Gene Expression Analysis and Histology}

Genes coding for proteins of the sarcomere and extracellular matrix were differentially expressed in the heart (Table 1). Thus, the myosin heavy chain alpha $(\alpha-M H C)$ and the myosin heavy chain beta $(\beta-M H C)$ were upregulated. Further, the collagen type I maintaining tissue structure and collagen type IV, a component of basal lamina that allows interaction with integrins and favors cell
A

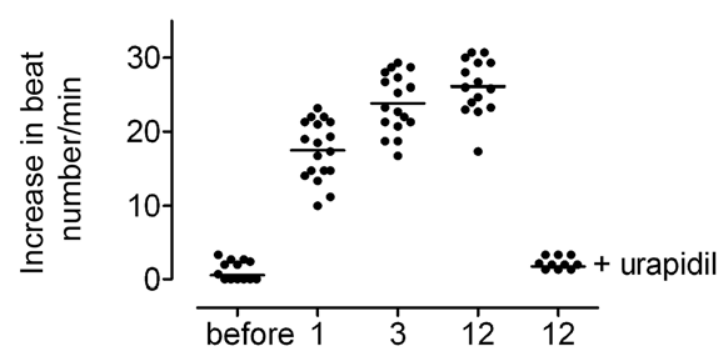

C

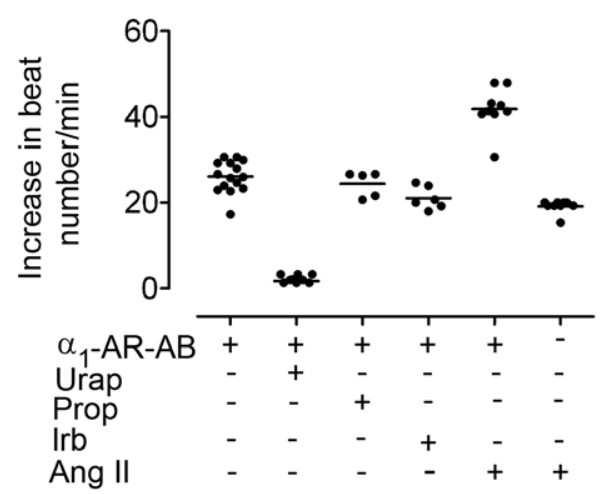

B

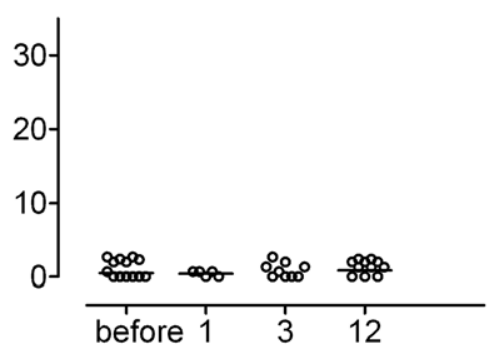

D

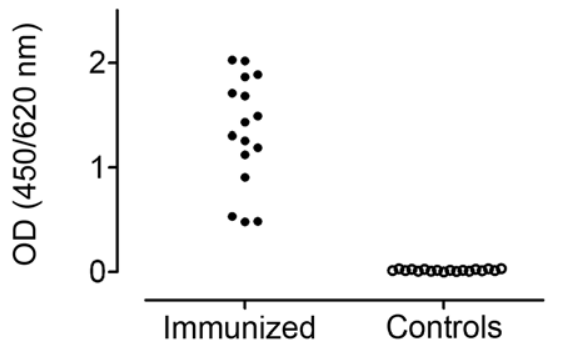

Figure 1. Rat $\alpha_{1 \mathbf{A}}-\mathbf{A R}-\mathbf{A B}$ detection. (A and B) Ordinate shows neonatal rat cardiomyocyte spontaneous beating rate; abscissa shows the immunization time point. IgGs from immunized rats increased the beating rate (A), compared to controls (B) Specificity was checked by inhibition with $1 \mu \mathrm{M}$ urapidil (Urap). (C) We excluded autoantibodies against other receptors using the b1-AR antagonist propranolol (Prop) and the AT1 receptor antagonist irbersartan (Irb). The combination of $\alpha_{1 A^{-}}-A R-A B$ and Ang II increased the cardiomyocyte contraction. (D) A high titer of $\alpha_{1 A^{-}} A R-A B$ was detected by ELISA in immunized rats, compared to controls.

doi:10.1371/journal.pone.0009409.g001 
A

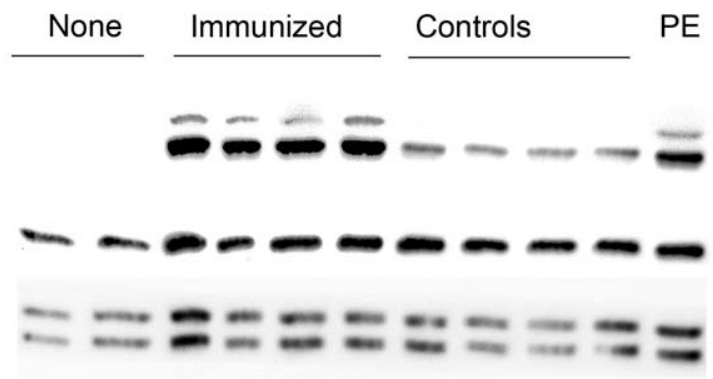

B

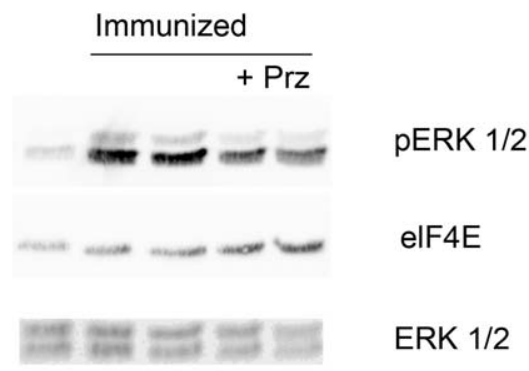

Figure 2. ERK1/2 activation in $\mathrm{CHO} / \alpha_{1 \mathrm{~A}}$-AR cells. (A) Activation after incubation with $\lg$ from immunized and control rats or with phenylephrine $(10 \mu \mathrm{M})$ for $10 \mathrm{~min}$ is shown. Lane 1 and 2 represent untreated cells. (B) The specificity of ERK1/2 activation by $\alpha_{1 \mathrm{~A}}-\mathrm{AR}-\mathrm{AB}$ was checked by prazosin inhibition (Prz, $1 \mu \mathrm{M})$. Lane 1 represents untreated cells. Eukaryotic initiation factor 4E (elF4E) and ERK1/2 antibody were used as loading control.

doi:10.1371/journal.pone.0009409.g002

adhesion, were increased expressed. The collagen IV binding glycoprotein laminin was also upregulated. Other genes coding for proteins involved in the $\mathrm{Ca}^{2+}$ signaling were represented. Among them were the cardiac ryanodine receptor 2 (Ryr2), the cardiac ATPase $2 \mathrm{Ca}^{2+}$-transporting, slow-twitch (Atp2a2), and the L-type calcium channel (Cacna1c). Important components of the energy metabolism like muscle glycogen phosphorylase and the peroxisome proliferators-activated receptor-gamma, co-activator 1 , alpha (Ppargc1a) a master regulator of metabolic function responsible for fatty acid uptake and oxidation and oxidative phosphorylation[10] were upregulated (Table 1).

The gene expression results were validated by TaqMan analysis for $\alpha-M H C($ Fold change $(\mathrm{FC})=3.5), \beta-M H C(\mathrm{FC}=2.8)$, collagen, type $\mathrm{I}$, alpha $1(\mathrm{FC}=1.7)$, and Cacnalc $(\mathrm{FC}=2.0)$. The cardiac expression of $\alpha_{1 \mathrm{~A}^{-}}, \alpha_{1 \mathrm{~B}^{-}}$or $\alpha_{1 \mathrm{D}^{-}} \mathrm{AR}$ subtype was not differential in immunized and control rats one year after immunization. Sirius red and Trichrome-Masson-Goldner staining were used for fibrosis detection. More fibrosis occurred in immunized rats
A
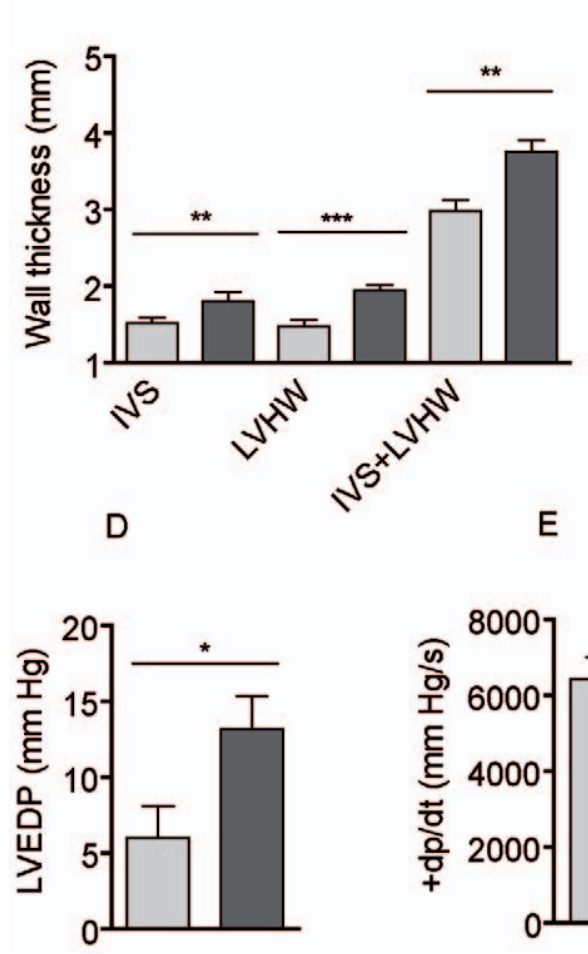

B

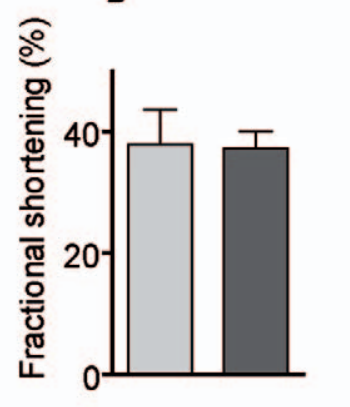

C

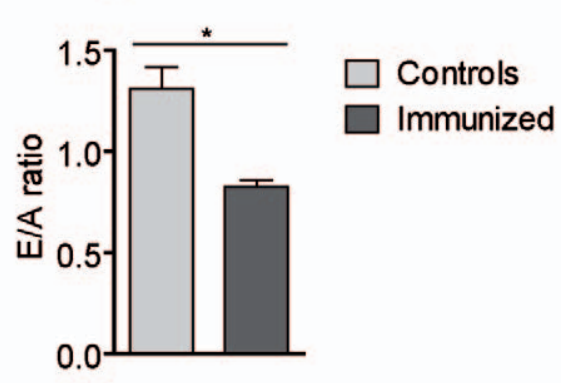

Figure 3. Echocardiography and invasive hemodynamic by conductance catheter at 12 months after first immunization. (A) Increased interventricular septum (IVS) and left ventricular heart wall (LVHW) thickness indicates hypertrophy. (B) Unchanged fractional shortening was a sign for preserved systolic function. (C) A decreased E/A ratio in immunized rats indicated a diastolic dysfunction. (D) Immunized rats had increased left ventricular end-diastolic pressure (LVEDP), (E) Unchanged dp/dt $\mathrm{max}_{\text {ax }}$ and (F) diminished dp/dt $\mathrm{min}_{\text {in }}$ indicating impaired relaxation/ diastolic function. (G) Mean diameter of cardiomyocytes showed a significant hypertrophy, 12 months after immunization with $\alpha_{1 A^{-}}-A R$ peptide compared to controls. ${ }^{*}$ indicates $\mathrm{P}<0.05$, ** indicate $\mathrm{P}<0.01,{ }^{* * *}$ indicate $\mathrm{P}<0.001$.

doi:10.1371/journal.pone.0009409.g003 


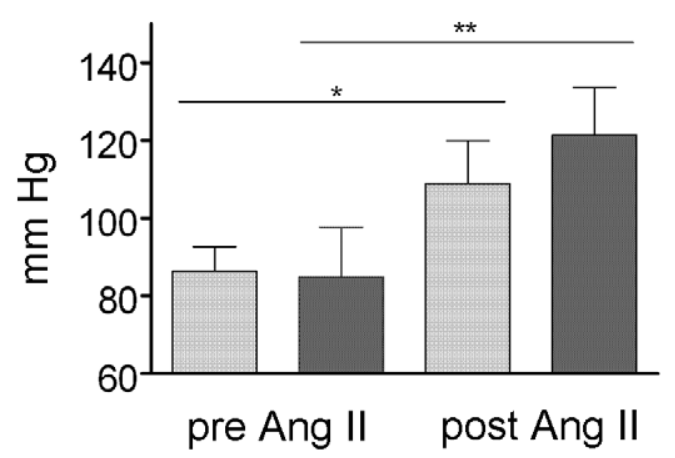

B

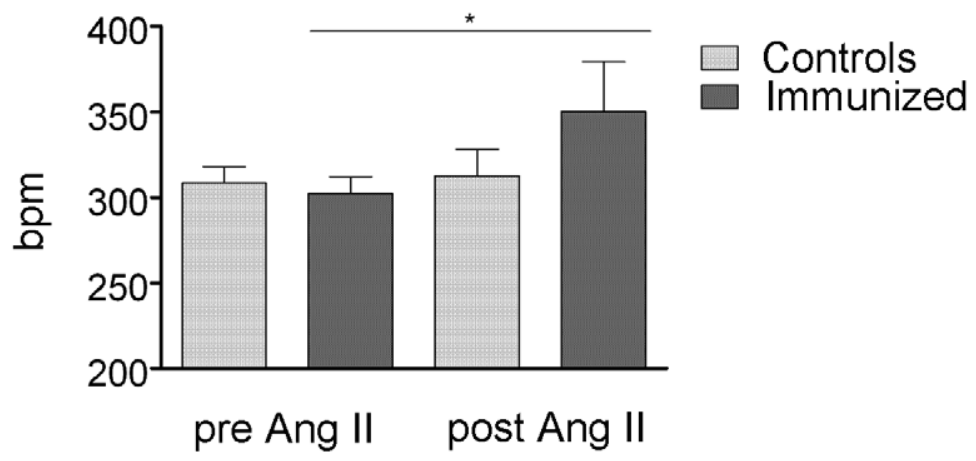

Figure 4. Mean arterial blood pressure (MAP) and heart rate 12 months after first immunization, pre and post angiotensin (Ang) II treatment. $\alpha_{1 A}-A R-A B$ in combination with the stressor Ang II cause a greater increase in blood pressure (A) and heart rate (B) in the immunized rats compared to the Ang II-treated controls. ${ }^{*}$ indicates $\mathrm{P}<0.05,{ }^{* *}$ indicate $\mathrm{P}<0.01$. doi:10.1371/journal.pone.0009409.g004

compared to the controls (Figure $5 \mathrm{~A}, \mathrm{~B}$ ). Perivascular fibrosis was significantly increased by $\alpha_{1 \mathrm{~A}}-\mathrm{AR}-\mathrm{AB}$, whereas interstitial fibrosis was significantly induced in combination with Ang II infusion (Figure 6).

\section{Ang II Effects in Immunized and Control Rats}

We tested whether or not immunized rats react more strongly to Ang II. Ang II further increased the chronotropic response of $\alpha_{1 \mathrm{~A}^{-}}$ $\mathrm{AR}-\mathrm{AB}$, whereas phenylephrine did not result in an incremental increase in the bioassay (Supplementary figure S1). The combination of $\alpha_{1 \mathrm{~A}}-\mathrm{AR}-\mathrm{AB}$ and Ang II caused a stronger increase in MAP in immunized rats, compared to the Ang II treated

Table 1. Selected differentially expressed genes in hearts of immunized rats.

\begin{tabular}{|c|c|c|}
\hline Gene & Ref.-Sequence & Fold \\
\hline \multicolumn{3}{|l|}{$\begin{array}{l}\text { change } \\
\text { Sarcomere }\end{array}$} \\
\hline Myosin heavy chain, alpha & NM_017239 & 2.7 \\
\hline Myosin heavy chain, beta & NM_017240 & 2.3 \\
\hline \multicolumn{3}{|l|}{ Collagens and extracellular matrix proteins } \\
\hline Collagen, type I, alpha 1 & NM_053304 & 1.5 \\
\hline Collagen, type I, alpha 2 & NM_053356 & 1.6 \\
\hline Procollagen, type IV, alpha 1 & XM_001067473 & 1.7 \\
\hline Procollagen, type IV, alpha 2 & XM_001076134 & 1.5 \\
\hline Laminin, gamma 1 & XM_001071300 & 1.9 \\
\hline \multicolumn{3}{|l|}{$\underline{\mathrm{Ca}^{2+} \text {-regulation }}$} \\
\hline Ryanodine receptor 2 , cardiac & XR_006681 & 1.7 \\
\hline $\begin{array}{l}\text { ATPase, } \mathrm{Ca}^{2+} \text {-transporting, cardiac muscle, slow } \\
\text { twitch } 2\end{array}$ & NM_001110139 & 1.5 \\
\hline $\mathrm{Ca}^{2+}$-channel, voltage-dependent, $\mathrm{L}$ type, alpha $1 \mathrm{C}$ & NM_012517 & 1.5 \\
\hline \multicolumn{3}{|l|}{ Energy metabolism } \\
\hline $\begin{array}{l}\text { Peroxisome proliferators-activated receptor-gamma, } \\
\text { coactivator } 1 \text {, alpha }\end{array}$ & NM_031347 & 1.8 \\
\hline Muscle glycogen phosphorylase & NM_012638 & 1.7 \\
\hline Phosphofructokinase, muscle & NM_031715 & 1.5 \\
\hline
\end{tabular}

controls (Figure 4, $\mathrm{P}=0.0339$ ). The ratio of heart/body weight $(\mathrm{HW} / \mathrm{BW} \times 1000)$ was significantly increased in the immunized rats compared to the controls $(2.31 \pm 0.13$ vs. 2.08 \pm 0.12 , $\mathrm{P}=0.0109$, Supplementary table S2). We used TrichromeMasson-Goldner staining to quantify our results and found that immunized rats developed more severe fibrosis than control rats (Figure $5 \mathrm{~A}, \mathrm{~B}$ ). The extent of fibrosis as a percent of tissue area was significant higher in the immunized rats $(14.4 \pm 4.54 \%$ vs. $8.90 \pm 2.31 \%$ in controls, $\mathrm{P}<0.00001)$. Ang II also induced a significant myocyte hypertrophy, which was not potentiated by $\alpha_{1 \mathrm{~A}}-\mathrm{AR}-\mathrm{AB}$ (data not shown).

\section{Discussion}

The important findings in our study were that active immunization results in production of activating antibodies to the $\alpha_{1 \mathrm{~A}}-\mathrm{AR}$, namely $\alpha_{1 \mathrm{~A}}-\mathrm{AR}-\mathrm{AB}$. The $\alpha_{1 \mathrm{~A}}-\mathrm{AR}-\mathrm{AB}$ in our model did not increase telemetrically measured blood pressure. However, they clearly caused cardiac target organ damage and resulted in a model of diastolic dysfunction. Finally, stimulation of the system with an additional stressor (Ang II) resulted in worsened targetorgan damage in the $\alpha_{1 \mathrm{~A}}-\mathrm{AR}-\mathrm{AB}$-producing animals compared to controls. The immediate question is, "how do the results reported here differ from those of Zhou et al [7]?" We relied on a different immunization sequence; our sequence corresponded to $\alpha_{1 \mathrm{~A}} \mathrm{AR}$, while Zhou et al used a sequence corresponding to $\alpha_{1 \mathrm{D}}-\mathrm{AR}$. These receptors are different. We investigated Ang II provocations, while they did not. We focused on target-organ damage. Finally, we monitored endorgan damage with echocardiography, invasive hemodynamics, histological analysis, radiotelemetrically determined blood pressure measurements, and by gene expression array.

The immunized rats developed specific $\alpha_{1 \mathrm{~A}} \mathrm{AR}-\mathrm{AB}$ that we detected by both cardiomyocyte contraction assay and ELISA. The blocking experiments underscored the specificity of these antibodies. The antibodies were capable of initiating $\alpha_{1 \mathrm{~A}}$-AR signaling as documented by our phospho-ERK1/2 experiments. We documented clear-cut differences in cardiac function by two independent techniques, one relying on direct cardiac catheterization that revealed a diastolic pathology. Cardiac structure was substantially different between the groups, as shown by echocardiography and by light microscopy. Our notion that a much more sensitive blood pressure measurement than the tail-cuff technique would show a difference in blood pressure between groups, proved 
A

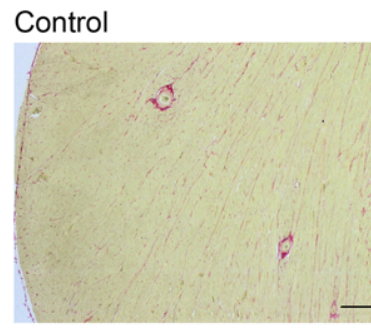

Immunized

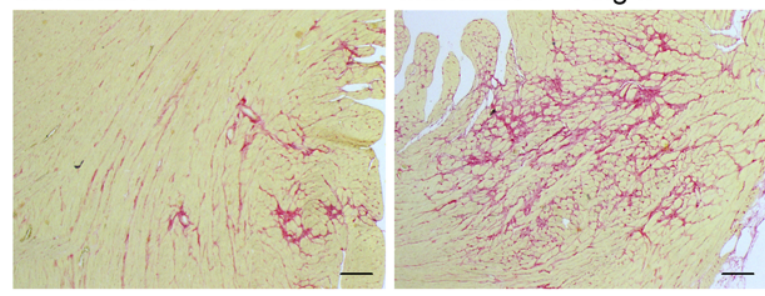

B

\section{Control}
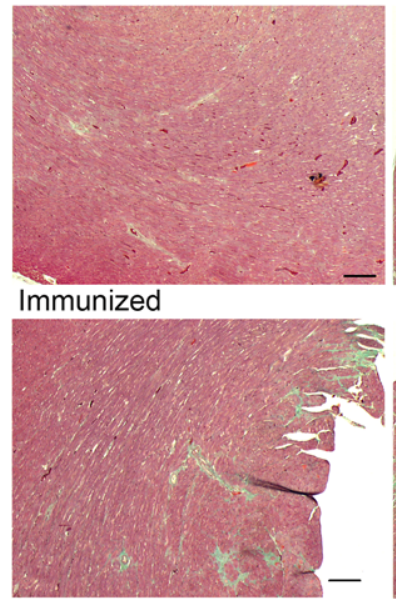

Control + Ang II

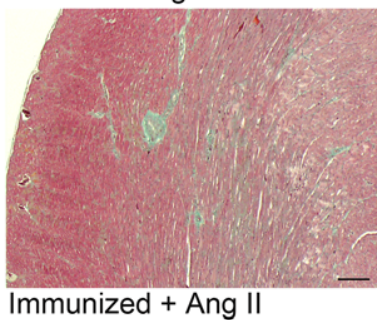

Immunized + Ang II

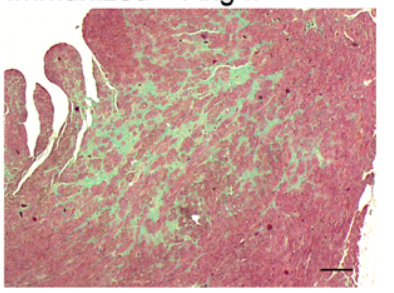

Figure 5. Cardiac remodeling and fibrosis in immunized rats, pre and post Ang II treatment. (A) Sirius red and (B) TrichromeMasson-Goldner staining. Both stains showed marked fibrosis in the hearts of immunized rats. $\alpha_{1 A}-A R-A B$, in combination with the stressor Ang II cause a significantly higher increase of fibrosis in immunized rat hearts compared to the Ang II treated controls (Bar $=0.2 \mathrm{~mm}$ ). doi:10.1371/journal.pone.0009409.g005

not to be the case. Nonetheless, the Ang II experiments showed clearly that when a driving force for hypertension is applied, the presence of $\alpha_{1 \mathrm{~A}}-\mathrm{AR}-\mathrm{AB}$ clearly aggravates target organ damage and blood pressure. Finally, we explored new mechanistic pathways.

Our gene expression analyses showed different compensatory mechanisms in structure and metabolism to maintain the cardiac function. In contrast to the failing rat heart with a shift in myosin isoform from $\alpha$ to $\beta$-MHC [11], we found instead an increased expression of both MHC isoforms. Up-regulation of $\beta$-MHC transcription can serve as an early and sensitive marker of cardiac hypertrophy [11,12] and may conserve energy [13,14]. Forced expression of $\alpha$-MHC may be beneficial in terms of increasing the myocardial contractility and may result in cardioprotection $[15,16]$.

Myocardial remodeling implies an alteration in the extracellular matrix composition and distribution. Accordingly, we found in our array analysis the upregulation of collagen type I and IV in immunized rat hearts. Collagen I and III maintain the tissue structure, transmit forces throughout the myocardium and contribute to the elastic properties of the myocardium [17]. The increased accumulation of collagen I and III has been associated mostly with fibrosis [18,19]. Type IV and VI collagens are components of the basal lamina and favors cell adhesion. The increased expression could be involved in the alteration of extracellular matrix cell interaction [20]. In human dilated cardiomyopathy, collagen is degraded by metalloproteinases and is replaced by fibrous intercellular deposits [21]. Zhou et al found increased MMP2 expression and activity in their $\alpha_{1 \mathrm{D}}-\mathrm{AR}-\mathrm{AB}$ immunization study [7]. We did not observe any increased metalloproteinase or tissue inhibitors of metalloproteinases. Other up-regulated genes encode molecular regulators of energy metabolism. The peroxisome proliferator-activated receptor gamma (PPAR- $\gamma$ ) coactivator 1-alpha (Ppargcla) activates multiple genes that are responsible for fatty acid uptake and oxidation and for oxidative phosphorylation [10]. The development of heart failure is accelerated by Ppargcla deficiency [22], suggesting that this coactivator may have a cardioprotective function.

Another important observation is the fact that genes coding for important $\mathrm{Ca}^{2+}$ regulating proteins, such as ATPase, $\mathrm{Ca}^{2+}$ transporting, slow-twitch (Atp2a2) coding for the sarcoplasmic/ endoplasmic reticulum calcium ATPase (SERCA2a), the cardiac ryanodine receptor 2, and the L-type calcium channel, alpha $1 \mathrm{C}$ subunit (Cacnac1c) were all up-regulated. The overexpression of SERCA2a in diseased hearts has been shown to result in the recovery of contractility [23,24,25] and in improved survival, corresponding with an improvement in energy consumption [26]. Furthermore, SERCA overexpression decreases or prevents cardiac hypertrophy $[27,28,29]$. In our earlier study we found that acute administration of purified $\alpha_{1}$-AR-autoantibodies from patients or rabbit $\alpha_{1 \mathrm{~A}}-\mathrm{AR}-\mathrm{AB}$ to neonatal cardiomyocytes affected intracellular $\mathrm{Ca}^{2+}$ at two different levels, namely the acute, shortterm elevation of intracellular $\mathrm{Ca}^{2+}$, and the increased transcript expression of Cacnalc [5]. In this study, we also found a long-term upregulation of Cacna1c. A link between increased L-type calcium channel, alpha $1 \mathrm{C}$ subunit levels and hypertrophy has also been demonstrated for the human heart [30].

Rysa et al. performed DNA microarray analysis in 12 monthold SHR with manifest hypertrophy, compared to 16-20 monthold SHR with diastolic dysfunction and transition to heart failure [31]. Most of the enhanced genes upregulated in the development of diastolic heart failure encoded for ECM proteins. ECM proteins were also upregulated in our study. However, whereas we found dysregulated transcripts for calcium homeostasis, myofilament contractile proteins, and cardiomyocyte cytoskeleton proteins, these pathways seemed not to play a significant role in the development of diastolic heart failure caused by pressure-overload hypertrophy. The two models and the experimental settings were considerably different. Our $\alpha_{1 \mathrm{~A}}$-AR-immunized rats were only 12 months old, had no hypertension, and no signs of heart failure, whereas the SHR rats were older and had signs of pressureinduced diastolic heart failure. Interestingly, Wallukat et al have shown that SHR develop autoantibodies against the $\beta 1$ adrenergic receptor that permanently stimulate the receptor, while $\alpha_{1}-\mathrm{AR}-\mathrm{AB}$ have not been found [32,33].

The $\alpha_{1}$-adrenergic receptors are important to both developmental cardiomyocyte growth and pathological hypertrophy. The $\alpha_{1 \mathrm{~A}^{-}}$ $\mathrm{AR}-\mathrm{AB}$ production induced hypertrophy by causing fibrosis and cardiomyocyte hypertrophy. Patel et al [34] showed that a 28-day infusion of a subpressor norepinephrine dose induced hypertrophy only by stimulating myocyte growth. No fibrosis or signs of diastolic dysfunction was present in their 28-day study. However, we 
A

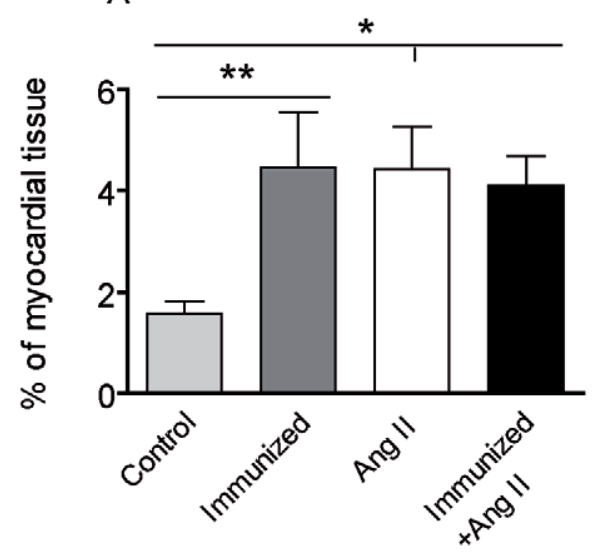

B

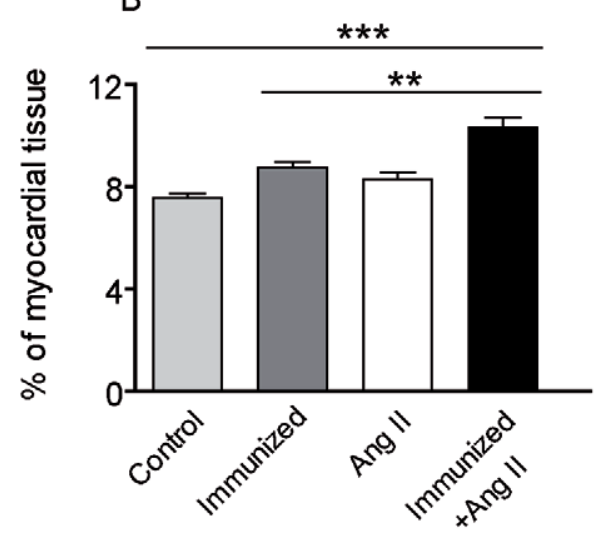

Figure 6. Myocardial perivascular and interstitial collagen. (A) Perivascular fibrosis was significantly increased in all three treated groups compared to control rats. (B) Interstitial fibrosis was significantly increased in immunized rats treated with Ang II when compared to controls, rats treated with Ang II or immunized rats. doi:10.1371/journal.pone.0009409.g006

investigated our model for 1 year. Du et al [35] reported that animals transgenic for the $\alpha_{1 \mathrm{~A}}-\mathrm{AR}$ showed a greater increase in myocardial fibrosis post-myocardial infarction, compared to the non-transgenic control animals. Transgenic lines with an even higher expression level developed progressive cardiac fibrosis.

In spite of subtype-selective agonists and antagonists and gene knockout and transgenic overexpression approaches, the question of which $\alpha_{1}$-AR subtype is involved predominantly in vasoconstrictive responses to sympathomimetic agonists has not been answered. The studies with knockout mice indicate that all subtypes play a role in the blood pressure response to $\alpha_{1}$-agonists and that the dominant contractile $\alpha_{1}-\mathrm{AR}$ is different in different vascular beds. Although we did not observe an increase in blood pressure by $\alpha_{1 \mathrm{~A}}-\mathrm{AR}-\mathrm{AB}$, our results do not justify the conclusion that the $\alpha_{1 \mathrm{~A}}-\mathrm{AR}$ is not involved in the blood pressure control. Our results are in line with results reported by Tanoue et al [36]. These investigators found a major role for $\alpha_{1 \mathrm{~A}}$-AR in maintaining basal blood pressure, whereas other subtypes such as $\alpha_{1 \mathrm{~B}}-\mathrm{AR}$ and $\alpha_{1 \mathrm{D}}-\mathrm{AR}$ were more important in the pressor response to catecholamines.

Our immunized $\alpha_{1}$-AR-AB-producing rats developed an increased LVEDP and diminished $\mathrm{dp} / \mathrm{dt}_{\min }$ in the face of preserved ejection fraction and fractional shortening. This stateof-affairs is termed "diastolic dysfunction" and is a precursor for diastolic heart failure. Half the patients with heart failure fall into this category, notably older women. The prognosis of the condition is no better, if not worse, than systolic heart failure. All medication trials to date have been disappointing. The $\alpha_{1}$-AR blockers have not been studied in detail in the context of diastolic dysfunction. Two smaller studies reported positive effects of $\alpha_{1}$-AR blockers on echocardiographic parameters of diastolic dysfunction. To our knowledge, this experimental model is the first to show that $\alpha_{1 \mathrm{~A}} \mathrm{AR}$ receptor stimulation can cause diastolic dysfunction independent of any change in blood pressure. De Blois et al showed that chronic $\alpha_{1}$-AR stimulation increases smooth muscle cell DNA replication is in arterial wall, leading to remodeling after vascular injury [37]. We speculate that alterations in peripheral resistance (pressure-overload) could have been responsible for the diastolic dysfunction we observed. The fact that we were unable to detect any blood pressure increases even with radiotelemetry, suggests the possibility that altered blood pressure buffering played a role. We demonstrated heart muscle cell (cardiomyocyte) hypertrophy, in addition to an increased cardiac fibrosis in the $\alpha_{1 \mathrm{~A}}-\mathrm{AR}-\mathrm{AB}$ model. We believe that these changes, independent of any blood pressure changes we were able to detect, resulted in the diastolic dysfunction that we observed.

Our Ang II experiments showed that Ang II markedly aggravated the already-present cardiac fibrosis. We used Ang II as a stimulus to further induce vascular dysfunction. The sympathetic nervous system and renin-angiotensin-aldosterone system act synergistically to elevate or maintain blood pressure. Ang II signaling plays a critical role in modulating many of the stimuli and signals that govern arterial aging, arterial structural, and vascular functional and adaptational responses. Ang II also potentiated the chronotropic response to $\alpha_{1 \mathrm{~A}}-\mathrm{AR}-\mathrm{AB}$, whereas phenylephrine infusion, as reported by Patel et al, did not [22].

Limitations in our study are the fact that we did not include a long-term treated control group, namely immunized rats producing $\alpha_{1 \mathrm{~A}}-\mathrm{AR}-\mathrm{AB}$ treated with chronic $\alpha_{1}$-AR blocker therapy. An additional desirable control group could consist of chronic phenylephrine infusion (for 1 year). Acute infusion experiments could elucidate the issue of baroreceptor reflex blood pressure buffering capacity or resetting that remains unanswered from our study. However, our acute experiments showed that the effects of $\alpha_{1 \mathrm{~A}}-\mathrm{AR}-\mathrm{AB}$ could be blocked pharmacologically. Chronic experiments could have allowed us to speculate with greater confidence on a possible role of $\alpha_{1}$-AR blockade to alleviate diastolic heart dysfunction and remodeling.

\section{Perspectives}

We elucidated agonistic autoimmunity-induced target-organ damage and showed that $\alpha_{1 \mathrm{~A}}-\mathrm{AR}-\mathrm{AB}$ after immunization caused diastolic dysfunction. Our animal model suggests that $\alpha_{1 \mathrm{~A}}-\mathrm{AR}-\mathrm{AB}$ could play a role in target-organ damage; however $\alpha_{1 \mathrm{~A}}-\mathrm{AR}-\mathrm{AB}$ are probably not initiators of hypertension. These findings have implications for the notion of viewing agonistic autoantibodies as primary treatment targets in human diseases [38]. Our findings also have implications concerning $\alpha_{1}$-AR blocker therapy. These agents may warrant a closer look, particularly in terms of diastolic dysfunction.

\section{Supporting Information}

Figure S1 Cardiomyocyte contraction assay. The incubation of cardiomyocytes with $\hat{\mathrm{I}} \pm_{1 \mathrm{~A}} \mathrm{AR}$-antibodies $(\mathrm{AB})$ or phenylephrine 
$(\mathrm{PE})$ caused an increase in cardiomyocyte contraction. This effect was not further potentiated by the combination of $\mathrm{AB}$ and PE. The combination of $\mathrm{AB}$ and Ang II resulted in a further raise of contraction.

Found at: doi:10.1371/journal.pone.0009409.s001 (0.13 MB DOC)

Table S1 Primer and probe sequences used for TaqMan RTPCR.

Found at: doi:10.1371/journal.pone.0009409.s002 (0.05 MB DOC)

Table S2 Physiological parameters of immunized and control rats.

Found at: doi:10.1371/journal.pone.0009409.s003 (0.03 MB DOC)

Table S3 Echocardiography of immunized and control rats 12 months after first immunization and after Ang II treatment.

\section{References}

1. Piascik MT, Perez DM (2001) Alphal-adrenergic receptors: new insights and directions. J Pharmacol Exp Ther 298: 403-410.

2. Fu ML, Herlitz H, Wallukat G, Hilme E, Hedner T, et al. (1994) Functional autoimmune epitope on alpha 1-adrenergic receptors in patients with malignant hypertension. Lancet 344: 1660-1663.

3. Liao YH, Wei YM, Wang M, Wang ZH, Yuan HT, et al. (2002) Autoantibodies against AT1-receptor and alphal-adrenergic receptor in patients with hypertension. Hypertens Res 25: 641-646.

4. Luther HP, Homuth V, Wallukat G (1997) Alpha 1-adrenergic receptor antibodies in patients with primary hypertension. Hypertension 29: 678-682.

5. Wenzel K, Haase H, Wallukat G, Derer W, Bartel S, et al. (2008) Potential relevance of alpha(1)-adrenergic receptor autoantibodies in refractory hypertension. PLoS ONE 3: e3742.

6. Jahns R, Boivin V, Hein L, Triebel S, Angermann CE, et al. (2004) Direct evidence for a beta 1-adrenergic receptor-directed autoimmune attack as a cause of idiopathic dilated cardiomyopathy. J Clin Invest 113: 1419-1429.

7. Zhou Z, Liao YH, Wei Y, Wei F, Wang B, et al. (2005) Cardiac remodeling after long-term stimulation by antibodies against the alphal-adrenergic receptor in rats. Clin Immunol 114: 164-173.

8. Brockway BP, Mills PA, Azar SH (1991) A new method for continuous chronic measurement and recording of blood pressure, heart rate and activity in the rat via radio-telemetry. Clin Exp Hypertens A 13: 885-895.

9. van Heerebeek L, Borbely A, Niessen HW, Bronzwaer JG, van der Velden J, et al. (2006) Myocardial structure and function differ in systolic and diastolic heart failure. Circulation 113: 1966-1973.

10. Huss JM, Kelly DP (2004) Nuclear receptor signaling and cardiac energetics. Circ Res 95: 568-578.

11. Mercadier JJ, Lompre AM, Wisnewsky C, Samuel JL, Bercovici J, et al. (1981) Myosin isoenzyme changes in several models of rat cardiac hypertrophy. Circ Res 49: 525-532.

12. Jones WK, Grupp IL, Doetschman T, Grupp G, Osinska H, et al. (1996) Ablation of the murine alpha myosin heavy chain gene leads to dosage effects and functional deficits in the heart. J Clin Invest 98: 1906-1917.

13. Holubarsch C, Goulette RP, Litten RZ, Martin BJ, Mulieri LA, et al. (1985) The economy of isometric force development, myosin isoenzyme pattern and myofibrillar ATPase activity in normal and hypothyroid rat myocardium. Circ Res 56: 78-86.

14. Sugiura S, Kobayakawa N, Fujita H, Yamashita H, Momomura S, et al. (1998) Comparison of unitary displacements and forces between 2 cardiac myosin isoforms by the optical trap technique: molecular basis for cardiac adaptation. Circ Res 82: 1029-1034.

15. Abraham WT, Gilbert EM, Lowes BD, Minobe WA, Larrabee P, et al. (2002) Coordinate changes in Myosin heavy chain isoform gene expression are selectively associated with alterations in dilated cardiomyopathy phenotype. Mol Med 8: 750-760.

16. James J, Martin L, Krenz M, Quatman C, Jones F, et al. (2005) Forced expression of alpha-myosin heavy chain in the rabbit ventricle results in cardioprotection under cardiomyopathic conditions. Circulation 111: 2339-2346.

17. Bishop JE (1998) Regulation of cardiovascular collagen deposition by mechanical forces. Mol Med Today 4: 69-75.

18. Weber KT, Sun Y, Tyagi SC, Cleutjens JP (1994) Collagen network of the myocardium: function, structural remodeling and regulatory mechanisms. J Mol Cell Cardiol 26: 279-292.

19. Villarreal FJ, Dillmann WH (1992) Cardiac hypertrophy-induced changes in mRNA levels for TGF-beta 1, fibronectin, and collagen. Am J Physiol 262: H1861-1866.
Found at: doi:10.1371/journal.pone.0009409.s004 (0.04 MB DOC)

\section{Acknowledgments}

We thank Sabine Bartel, Jutta Meisel, Reika Langanki, Astrid Schiche, Ralph Plehm, Melanie Soosten, Juliane Anders and May-Britt Köhler for the excellent technical assistance. Martin Michel, Dept. Pharmacology \& Pharmacotherapy, University of Amsterdam, Netherlands provided CHO cells stably transfected with human $\alpha_{1 \mathrm{~A}}-\mathrm{AR}$. We thank Hannelore Haase for critical reading of the manuscript.

\section{Author Contributions}

Conceived and designed the experiments: KW GW DNM RD RD. Performed the experiments: KW GW FQ HS FH AH RF HH. Analyzed the data: KW FQ NH HS OH FH AH RF HH FCL DNM RD. Contributed reagents/materials/analysis tools: KW GW FQ NH HS OH HH RD RD. Wrote the paper: KW FCL DNM RD.
20. Chapman D, Weber KT, Eghbali M (1990) Regulation of fibrillar collagen types I and III and basement membrane type IV collagen gene expression in pressure overloaded rat myocardium. Circ Res 67: 787-794.

21. Gunja-Smith Z, Morales AR, Romanelli R, Woessner JF, Jr. (1996) Remodeling of human myocardial collagen in idiopathic dilated cardiomyopathy. Role of metalloproteinases and pyridinoline cross-links. Am J Pathol 148: 1639-1648.

22. Arany Z, Novikov M, Chin S, Ma Y, Rosenzweig A, et al. (2006) Transverse aortic constriction leads to accelerated heart failure in mice lacking PPARgamma coactivator lalpha. Proc Natl Acad Sci U S A 103: 10086-10091.

23. Schmidt U, del Monte F, Miyamoto MI, Matsui T, Gwathmey JK, et al. (2000) Restoration of diastolic function in senescent rat hearts through adenoviral gene transfer of sarcoplasmic reticulum $\mathrm{Ca}(2+)$-ATPase. Circulation 101: 790-796.

24. Miyamoto MI, del Monte F, Schmidt U, DiSalvo TS, Kang ZB, et al. (2000) Adenoviral gene transfer of SERCA2a improves left-ventricular function in aorticbanded rats in transition to heart failure. Proc Natl Acad Sci U S A 97: 793-798.

25. Kawase Y, Ly HQ, Prunier F, Lebeche D, Shi Y, et al. (2008) Reversal of cardiac dysfunction after long-term expression of SERCA2a by gene transfer in a pre-clinical model of heart failure. J Am Coll Cardiol 51: 1112-1119.

26. del Monte F, Williams E, Lebeche D, Schmidt U, Rosenzweig A, et al. (2001) Improvement in survival and cardiac metabolism after gene transfer of sarcoplasmic reticulum $\mathrm{Ca}(2+)$-ATPase in a rat model of heart failure. Circulation 104: 1424-1429.

27. Ito K, Yan X, Feng X, Manning WJ, Dillmann WH, et al. (2001) Transgenic expression of sarcoplasmic reticulum $\mathrm{Ca}(2+)$ atpase modifies the transition from hypertrophy to early heart failure. Circ Res 89: 422-429.

28. Sakata S, Lebeche D, Sakata Y, Sakata N, Chemaly ER et al. (2007) Transcoronary gene transfer of SERCA2a increases coronary blood flow and decreases cardiomyocyte size in a type 2 diabetic rat model. Am J Physiol Heart Circ Physiol 292: H1204-1207.

29. Nakayama H, Otsu K, Yamaguchi O, Nishida K, Date MO, et al. (2003) Cardiac-specific overexpression of a high Ca2+ affinity mutant of SERCA2a attenuates in vivo pressure overload cardiac hypertrophy. FASEB J 17: 61-63.

30. Haase H, Kresse A, Hohaus A, Schulte HD, Maier M, et al. (1996) Expression of calcium channel subunits in the normal and diseased human myocardium. J Mol Med 74: 99-104.

31. Rysa J, Leskinen H, Ilves M, Ruskoaho H (2005) Distinct upregulation of extracellular matrix genes in transition from hypertrophy to hypertensive heart failure. Hypertension 45: 927-933.

32. Wallukat G, Blasig IE, Morwinski R, Herrmann HJ, Rohde E (1995) The sera of spontaneously hypertensive rats contain agonistic auto-antibodies against the beta 1-adrenoceptor. J Hypertens 13: 1031-1036.

33. Wallukat G, Podlowski S, Nissen E, Morwinski R, Csonka C, et al. (2003) Functional and structural characterization of anti-betal-adrenoceptor autoantibodies of spontaneously hypertensive rats. Mol Cell Biochem 251: 67-75.

34. Patel MB, Stewart JM, Loud AV, Anversa P, Wang J, et al. (1991) Altered function and structure of the heart in dogs with chronic elevation in plasma norepinephrine. Circulation 84: 2091-2100.

35. Du XJ, Gao XM, Kiriazis H, Moore XL, Ming Z, et al. (2006) Transgenic alphalA-adrenergic activation limits post-infarct ventricular remodeling and dysfunction and improves survival. Cardiovasc Res 71: 735-743.

36. Tanoue A, Koshimizu TA, Tsujimoto G (2002) Transgenic studies of alpha(1)adrenergic receptor subtype function. Life Sci 71: 2207-2215.

37. deBlois D, Schwartz SM, van Kleef EM, Su JE, Griffin KA, et al. (1996) Chronic alpha 1-adrenoreceptor stimulation increases DNA synthesis in rat arterial wall. Modulation of responsiveness after vascular injury. Arterioscler Thromb Vasc Biol 16: 1122-1129.

38. Verlohren S, Muller DN, Luft FC, Dechend R (2009) Immunology in hypertension, preeclampsia, and target-organ damage. Hypertension 54: 439-443. 